\title{
TAYLOR-DIRICHLET SERIES AND ALGEBRAIC DIFFERENTIAL-DIFFERENCE EQUATIONS ${ }^{1}$
}

\author{
FRANK WADLEIGH
}

ABSTRACT. It is proved that if a convergent Taylor-Dirichlet series

$$
\sum_{k=0}^{\infty} P_{k}(s) e^{-\lambda_{k} s}, \quad s=\sigma+i t, \lambda_{k} \in \mathrm{C}, P_{k}(s) \in \mathrm{C}[s], \mathscr{R}\left(\lambda_{k}\right) \uparrow \infty,
$$

satisfies an algebraic differential-difference equation then the set of its exponents $\left\{\lambda_{k}\right\}_{k=0}^{\infty}$ has a finite, linear, integral basis. This generalizes a theorem of $\mathbf{A}$. Ostrowski.

An application of the theorem to a problem of oscillation in neuro-muscular systems is indicated.

1. Introduction. According to a well-known theorem of A. Ostrowski [7, Satz 6, p. 260] if a convergent Dirichlet series

$$
\sum_{k=0}^{\infty} a_{k} e^{-\lambda_{k} s}, \quad s=\sigma+i t, a_{k} \in \mathbf{C},
$$

with real exponents $\lambda_{0}<\lambda_{1}<\ldots \rightarrow \infty$ satisfies an algebraic differential-difference equation then the set of its exponents $\left\{\lambda_{k}\right\}_{k=0}^{\infty}$ has a finite, linear, integral basis.

In this paper we show that Ostrowski's theorem continues to hold for a convergent Taylor-Dirichlet series

$$
\phi(s)=\sum_{k=0}^{\infty} P_{k}(s) e^{-\lambda_{k} s}, \quad \lambda_{k} \in \mathbf{C}, P_{k}(s) \neq 0,
$$

where the $P_{k}$ are polynomials with complex coefficients and the real parts $\lambda_{k}^{\prime}$ of $\lambda_{k}$ satisfy $\lambda_{0}^{\prime}<\lambda_{1}^{\prime}<\ldots \rightarrow \infty$. The theorem is stated and proved in $\$ 3$. In $\$ 4$ we briefly indicate an area of application to neuro-muscular systems.

We recall that an algebraic differential-difference equation has the form

$$
G\left(x, f^{\left(m_{1}\right)}\left(x+h_{1}\right), \ldots, f^{\left(m_{r}\right)}\left(x+h_{r}\right)\right)=0
$$

where $G$ is a polynomial

$$
G\left(x, x_{1}, \ldots, x_{r}\right)=\Sigma C_{k_{1}, \ldots, k_{r}} x_{1}^{k_{1}} \ldots x_{r}^{k_{r}}
$$

with coefficients depending on $x$, and the ordered pairs $\left(h_{1}, m_{1}\right), \ldots,\left(h_{r}, m_{r}\right)$ of real numbers $h_{k}$ and nonnegative integers $m_{k}$ are distinct. By the total degree of $G$ is meant, as usual, the maximum value of the sums $k_{1}+\cdots+k_{r}$. For a discussion

Received by the editors February 28, 1978 and, in revised form, October 1, 1979.

AMS (MOS) subject classifications (1970). Primary 30A16; Secondary $34 J 10$.

${ }^{1}$ This work was performed while the author was associated with the National Research Institute for Mathematical Sciences, Pretoria, Republic of South Africa. 
of convergence of (1) refer to Valiron [10] where it is shown that if the degrees $\mu_{k}$ of $P_{k}(s)$ and the exponents $\lambda_{k}$ satisfy the conditions

$$
\lim _{k \rightarrow \infty} \frac{\mu_{k}}{\lambda_{k}}=0, \quad \lim _{k \rightarrow \infty} \frac{\log k}{\lambda_{k}}=0
$$

then the region of convergence of (1) is the same as that of the associated classical Dirichlet series $\Sigma_{1}^{\infty} A_{k} \exp \left(-s \lambda_{k}\right)$, where $A_{k}$ is the maximum of the moduli of the coefficients of $P_{k}$. Convergence of these latter series has been treated by a number of authors, for instance, Väisälä [9] and Miškelevičius [5]. Further results on Taylor-Dirichlet series can be found in Lepson [3], Lunc [4] and Blambert and Berland [1].

2. Lemmas. The proof of Ostrowski's theorem depends on three lemmas, the first of which [7, p. 246] states that an analytic function $g(s)$ which satisfies an algebraic differential-difference equation of the form (2) also satisfies an equation

$$
F\left(f^{\left(m_{1}\right)}\left(s+h_{1}\right), \ldots, f^{\left(m_{r}\right)}\left(s+h_{r}\right)\right)=0
$$

where the polynomial $F$ does not contain the variable $s$ as one of its arguments and where the total degree of $F$ does not exceed that of $G$. This result carries over without change to (1). Lemmas 1 and 2 which follow are modifications, respectively, of Ostrowski's Hilfssatz 2, p. 247 and Hilfssatz 3, p. 248 of [7].

LEMMA 1. The exponential polynomial

$$
E(\lambda)=\sum_{k=1}^{q} P_{k}(\lambda) e^{\alpha_{k} \lambda} \neq 0, \quad P_{k}(\lambda) \in \mathbf{C}[\lambda],
$$

where the exponents $\alpha_{k}$ are distinct real numbers has some zero-free right half-plane; i.e. there exists a positive number $B$ such that all zeros of $E(\lambda)$ have real part less than $B$.

Proof. Well known; see, e.g., Langer [2, p. 224].

Definition 1. A Taylor-Dirichlet series (1) is said to satisfy the algebraic differential-difference equation (3) formally if all coefficients of the series arising from formal substitution of (1) in the left-hand side of (3) vanish identically.

Definition 2. Throughout, the 'exponent' of a term of the form $P(s) e^{-\lambda s}$ will refer to the number $\lambda$.

We now state and prove the main lemma.

Lemma 2. If the Taylor-Dirichlet series (1) satisfies an algebraic differential-difference equation formally in which the variable $s$ does not occur explicitly then every exponent $\lambda_{k}$ of sufficiently large index $k$ can be expressed as a linear, integral combination of the preceding exponents $\lambda_{0}, \lambda_{1}, \ldots, \lambda_{k-1}$.

Proof. We prove the lemma for $0 \leqslant \lambda_{0}^{\prime}$ since the general situation is treated exactly as in [7]. By hypothesis, $\phi(s)$ satisfies an algebraic differential-difference equation of the form (3) formally. Let $N$ be the total degree of the polynomial $F$ and suppose that $\phi(s)$ does not satisfy formally any nontrivial algebraic differential-difference equation of total degree less than $N$. 
The partial derivatives

$$
F_{\rho}\left(f^{\left(m_{\nu}\right)}\left(s+h_{\nu}\right)\right)=\frac{\partial F\left(f^{\left(m_{\nu}\right)}\left(s+h_{\nu}\right)\right)}{\partial\left(f^{\left(m_{\rho}\right)}\left(s+h_{\rho}\right)\right)}
$$

where we have written $F\left(f^{\left(m_{n}\right)}\left(s+h_{\nu}\right)\right)$ in place of $F\left(f^{\left(m_{1}\right)}\left(s+h_{1}\right), \ldots\right.$, $\left.f^{\left(m_{r}\right)}\left(s+h_{r}\right)\right)$ are clearly polynomials in $f^{\left(m_{3}\right)}\left(s+h_{\nu}\right), \nu=1,2, \ldots, r$, of total degree less than $N$.

By the minimality of $N$, the expression $F_{\rho}\left(\phi^{\left(m_{v}\right)}\left(s+h_{\nu}\right)\right)$ does not vanish formally. We can write the first (ordered by real parts of exponents) nonzero term of this series in the form

$$
Q_{\rho}(s) e^{-\Lambda_{\rho} s}
$$

where $\Lambda_{\rho}$ is a linear, integral combination of a finite number of $\lambda_{k}$ 's and $Q_{\rho}(s)$ is a polynomial in $s$ with coefficients depending on these same $\lambda_{k}$ 's. Let $l_{0}=$ $\min _{1<\rho<r}\left\{\Lambda_{\rho}^{\prime}\right\}$ and suppose there are $m$ such terms so that $\Lambda_{\rho_{1}}^{\prime}=\cdots=\Lambda_{\rho_{m}}^{\prime}=l_{0}$ and that for all other terms of the form (4) we have $\Lambda_{\rho}^{\prime}>l_{0}$. Clearly only a finite number of terms of the series $\phi^{\left(m_{3}\right)}\left(s+h_{\nu}\right)$ contribute to the leading nonzero term (4) (or to the preceding terms which have cancelled). It follows from this and the monotonicity of the $\lambda_{k}^{\prime}$ that a positive number $l_{1}$ exists such that if

$$
\lambda_{n}^{\prime}>l_{1}
$$

then $\phi(s)$ can be split up into two sums

$$
\phi(s)=A_{n}(s)+R_{n}(s)
$$

where

$$
A_{n}(s)=\sum_{k=0}^{n-1} P_{k}(s) e^{-\lambda_{k} s}, \quad R_{n}(s)=\sum_{k=n}^{\infty} P_{k}(s) e^{-\lambda_{k} s}
$$

and the series $F_{\rho}\left(A_{n}^{\left(m_{s}\right)}\left(s+h_{\nu}\right)\right)$ will have the same leading nonzero term as $F_{\rho}\left(\phi^{\left(m_{\nu}\right)}\left(s+h_{\nu}\right)\right)$, namely (4).

Application of Taylor's formula for multivariate polynomials yields the finite expansion

$$
\begin{aligned}
F\left(\phi^{\left(m_{\nu}\right)}\left(s+h_{\nu}\right)\right)= & F\left(A_{n}^{\left(m_{\nu}\right)}\left(s+h_{\nu}\right)\right)+\sum_{1<\rho<r} F_{\rho}\left(A_{n}^{\left(m_{p}\right)}\left(s+h_{\nu}\right)\right) R_{n}^{\left(m_{\rho}\right)}\left(s+h_{\rho}\right) \\
& +\sum_{1<\rho<\eta<r} F_{\rho, \eta}\left(A_{n}^{\left(m_{p}\right)}\left(s+h_{\nu}\right)\right) R_{n}^{\left(m_{\rho}\right)}\left(s+h_{\rho}\right) R_{n}^{\left(m_{\eta}\right)}\left(s+h_{\eta}\right) \\
& +\ldots
\end{aligned}
$$

where $F_{\rho, \eta}$ denotes the second-order derivative apart from factorial coefficients.

Consider the third member on the right-hand side of (6) (reserving the word 'term' for series). A lower bound for the real part of the exponent of the first nonzero term of this expression as a formal Taylor-Dirichlet series is clearly $2 \lambda_{n}^{\prime}$, with corresponding lower bounds for the fourth, fifth, ... members on the righthand side of (6) being $3 \lambda_{n}^{\prime}$, $4 \lambda_{n}^{\prime}$, etc. 
In order to express $R_{n}^{\left(m_{\rho}\right)}(s)$ in a simple form we use the notation $D=d / d s$, the formal differential operator:

$$
R_{n}^{\left(m_{\rho}\right)}(s)=\frac{d^{\left(m_{\rho}\right)}}{d s^{m_{\rho}}}\left(\sum_{k=n}^{\infty} P_{k}(s) e^{-\lambda_{k} s}\right)=e^{-\lambda_{n} s}\left(D-\lambda_{n}\right)^{m_{\rho}}\left(P_{n}(s)\right)+\ldots
$$

We can now write the initial term of the second member on the right-hand side of (6) as

$$
e^{-\left(l_{0}+\lambda_{n}\right)} E\left(s, \lambda_{n}\right)
$$

where

$$
E(s, \lambda)=\sum_{j=1}^{m} Q_{\rho_{j}}(s)(D-\lambda)^{m_{\rho}}\left(P_{n}(s)\right) e^{-\lambda h_{\rho_{j}}}
$$

and is, for fixed $s$, an exponential polynomial of the kind considered in Lemma 1. Since the polynomials $Q_{\rho_{1}}, \ldots, Q_{\rho_{r}}$ and $P_{n}$ are $\not \equiv 0$ and the ordered pairs $\left(h_{\rho_{1}}, m_{\rho_{1}}\right), \ldots,\left(h_{\rho_{r}}, m_{\rho_{r}}\right)$ are distinct, there exists (at least) one value of $s$, say $s=s^{*}$ for which $E\left(s^{*}, \lambda\right) \not \equiv$ as a function of $\lambda$.

We may therefore apply Lemma 1 to $E\left(s^{*}, \lambda\right)$ and infer the existence of a positive number $l_{2}$ such that for all $\lambda_{n}$ whose real part $\lambda_{n}^{\prime}$ satisfies

$$
\lambda_{n}^{\prime}>l_{2}
$$

we have $E\left(s^{*}, \lambda_{n}\right) \neq 0$.

The term (7) therefore cannot cancel with the initial term of the third, fourth, ... members on the right-hand side of (6) provided $l_{0}+\lambda_{n}^{\prime}<2 \lambda_{n}^{\prime}$; that is, if

$$
\lambda_{n}^{\prime}>l_{0}
$$

Combining the above we see from (5), (8) and (9) that if we take $\lambda_{n}^{\prime}>l_{0}+l_{1}+l_{2}$ then (7) holds and the nonvanishing term (4) cannot be cancelled by any of the third, fourth, ... members on the right-hand side of (6). By hypothesis, however, each term of the series on the right-hand side of (6) must vanish; therefore $F\left(A_{n}^{\left(m_{p}\right)}\left(s+h_{\nu}\right)\right)$ must contain a term whose exponent is $l_{0}+\lambda_{n}$. Hence by the definition of $A_{n}(s), l_{0}+\lambda_{n}$ must be a linear, integral combination of $\lambda_{0}, \lambda_{1}, \ldots, \lambda_{n-1}$. The same is true for $l_{0}$ since $\lambda_{n}^{\prime}>l_{0}$, and hence we see that $\lambda_{n}$ itself is a linear, integral combination of the preceding exponents.

\section{Statement and proof of the theorem.}

THEOREM. If the convergent Taylor-Dirichlet series (1) satisfies an algebraic differential-difference equation then the set of its exponents $\left\{\lambda_{k}\right\}_{k=0}^{\infty}$ has a finite, linear, integral basis.

Proof. By the remarks at the beginning of $\$ 2$ it suffices to show that if $\phi(s)$ (defined by (1)) satisfies (3) then it also satisfies this equation formally, for then we may use Lemma 2 to complete the proof. In order to obtain the desired contradiction we therefore assume that $\phi(s)$ does not satisfy (3) formally. We can write the first (ordered, as before, according to real parts of exponents) nonzero term of the series obtained by formal substitution of $\phi\left(s+h_{\nu}\right)$ into the left-hand side of (3) as 


$$
Q(s) e^{-\Lambda s}
$$

where $\Lambda$ is a linear combination of a finite number of $\lambda_{k}$ 's and $Q(s)$ is a polynomial in $s$ whose coefficients depend on these $\lambda_{k}$ 's. We obviously have

$$
Q(s) \not 0 \text {. }
$$

We proceed to split $\phi(s)$ into two sums as in the proof of Lemma 2, $\phi(s)=A_{n}(s)+$ $R_{n}(s)$ where $n$ is taken so large that $\lambda_{n}^{\prime}>\Lambda^{\prime}$, thus ensuring that the first nonzero term of $F\left(A_{n}^{\left(m_{\nu}\right)}\left(s+h_{\nu}\right)\right)$ will also be $Q(s) e^{-\Lambda s}$.

Writing

$$
S_{n}(s)=P_{n}(s)+P_{n+1}(s) e^{-\left(\lambda_{n+1}-\lambda_{n}\right) s}+\ldots
$$

then

$$
\phi(s)=A_{n}(s)+e^{-\lambda_{n} s} S_{n}(s)
$$

and

$$
S_{n}(s)=O\left(s^{\mu_{n}}\right), \quad s \rightarrow \infty
$$

where $\mu_{n}=\operatorname{deg} P_{n}(s)$.

Here and subsequently all limits are taken as $s$ assumes real values. From (12) we get

$$
\phi^{\left(m_{\nu}\right)}\left(s+h_{\nu}\right)=A_{n}^{\left(m_{\nu}\right)}\left(s+h_{\nu}\right)+e^{-\lambda_{n} s} T_{n}^{\left(m_{\nu}\right)}\left(s, \lambda_{n}\right)
$$

where

$$
T_{n}^{\left(m_{v}\right)}\left(s, \lambda_{n}\right)=O\left(s^{\mu_{n}}\right), \quad s \rightarrow \infty .
$$

Replacing (12) and (13) into the left-hand side of (3) and applying Taylor's formula we have

$$
0=F\left(\phi^{\left(m_{\nu}\right)}\left(s+h_{\nu}\right)\right)=F\left(A_{n}^{\left(m_{\nu}\right)}\left(s+h_{\nu}\right)\right)+e^{-\lambda_{n} s} \Phi(s)
$$

where

$$
\Phi(s)=O\left(s^{M}\right), \quad s \rightarrow \infty,
$$

for some positive integer $M$.

On the other hand we can write

$$
F\left(A_{n}^{\left(m_{\nu}\right)}\left(s+h_{\nu}\right)\right)=e^{-\Lambda s}(Q(s)+h(s))
$$

with $h(s) \rightarrow 0$ as $s \rightarrow \infty$.

Thus if we insert the right-hand side of (17) in (15) we get

$$
0=e^{-\Lambda s}(Q(s)+h(s))+e^{-\lambda_{n} s} \Phi(s) .
$$

Multiplying (18) by $e^{\Lambda s}$ and taking the limit as $s \rightarrow \infty$ we obtain $Q(s) \equiv 0$ which contradicts (11) and proves the theorem.

4. An application. We indicate briefly how our theorem may be applied to a solution of the following algebraic differential-difference equation

$$
\sum_{k=0}^{4} a_{k} f^{(k)}(t)+\sum_{k=1}^{3}\left\{b_{k} f\left(t-t_{k}\right)+c_{k} f^{(1)}\left(t-t_{k}\right)\right\}=0
$$


occurring in the investigation of oscillations in neuro-muscular systems [6]. Here we write $f^{(0)}(t)$ for $f(t)$ and $f^{(1)}(t)$ for $f^{\prime}(t)$. The $a_{k}, b_{k}, c_{k}$ denote complex constants and $0<t_{1}<t_{2}<t_{3}$.

Substituting $f(t)=e^{s t}$ in (19) gives

$$
e^{t_{3} s} \sum_{k=0}^{4} a_{k} s^{k}+\sum_{k=1}^{3}\left(b_{k}+c_{k} s\right) e^{\left(t_{3}-t_{k}\right) s}=0
$$

for the transcendental characteristic equation. By a well-known theorem of Pontryagin [8] (20) has in general infinitely many roots $s$ with negative real part. There are, moreover, only finitely many multiple roots.

Denote the roots of (20) having negative real part by

$$
s_{k}=-p_{k}+i q_{k}, \quad p_{k}>0, k=1,2, \ldots,
$$

where $s_{k}$ has multiplicity $n_{k}$.

The corresponding particular solutions of (19) are then

$$
t^{\nu} e^{-p_{k} t} \cos q_{k} t, \quad t^{\nu} e^{-p_{k} t} \sin q_{k} t, \quad \nu=0,1, \ldots, n_{k}-1 .
$$

The general solution of (20) has the form

$$
f(t)=\sum_{k=1}^{\infty} e^{-P_{k} t}\left\{P_{k}(t) \cos q_{k} t+Q_{k}(t) \sin q_{k} t\right\}
$$

where $P_{k}(t)$ and $Q_{k}(t)$ are polynomials in $t$ of degree $n_{k}-1$ with complex coefficients such that (21) converges absolutely and uniformly for $t \geqslant 0$ and is four times differentiable.

Applying our theorem to the function $f(t)$ there exists then a finite set of numbers $p_{1}, p_{2}, \ldots, p_{n}$ such that

$$
f(t)=\sum_{k=1}^{\infty} e^{-\Lambda_{k} t}\left\{P_{k}(t) \cos q_{k} t+Q_{k}(t) \sin q_{k} t\right\}
$$

where $\Lambda_{k}=\sum_{j=1}^{n} \alpha_{k j} p_{j}$, for some integers $\alpha_{k j}$.

The application described above has served primarily as an example to illustrate the possible usefulness of the theorem. It is intended to pursue further such applications in a subsequent paper.

\section{REFERENCES}

1. M. Blambert and M. Berland, Sur la convergence des éléments LC-dirichlétiens, C. R. Acad. Sci. Paris Sér. A-B 281 (1975), A963-A966. MR 53\#3278.

2. R. E. Langer, On the zeros of exponential sums and integrals, Bull. Amer. Math. Soc. 37 (1931), 213-239.

3. B. Lepson, On hyperdirichlet series and on related questions of the general theory of functions, Trans. Amer. Math. Soc. 172 (1952), 18-45. MR13\#636.

4. G. Lunc, On series of Taylor-Dirichlet type, Izv. Akad. Nauk Armjan. SSR Ser. Fiz.-Mat. Nauk 14 (1961), no. 2, 7-16. MR 24\# A212. (Russian)

5. A. Miškelevičius, On the convergence of Dirichlet series, Litovsk. Mat. Sb. 3 (1963), no. 2, 105-113. MR 34\# 4466. (Russian)

6. M. N. Oguztöreli and R. B. Stein, An analysis of oscillations in neuro-muscular systems, J. Math. Biol. 2 (1975), 87-105.

7. A. Ostrowski, Über Dirichletsche Reihen und algebraische Differentialgleichungen, Math. Z. 8 (1921), 241-298. 
8. L. S. Pontryagin, On the zeros of some elementary transcendental functions, Izv. Akad. Nauk SSSR Ser. Mat. 6 (1942), 115-134.

9. K. Väisälä, Verallgemeinerung des Begriffes der Dirichletschen Reihen, Acta Univ. Dorp. AI 2 (1921), 3-32.

10. G. Valiron, Sur les solutions des équations différentielles linéaires d'ordre infini et à coéfficients constants, Ann. Ecole Norm. 46 (1929), 25-53.

National Research Institute for Mathematical Sciences, Pretoria, Republic of South Africa

Current address: Department of Bioengineering, University of California at San Diego, La Jolla, California 92093 